\title{
Quantitative analysis of diffusion weighted MR images of intracerebral haemorrhage by signal intensity gradient technique
}

\author{
Supriya S. Shanbhag1, ", Gururaj R. Udupi ${ }^{2}$, K. Mothiram Patil ${ }^{3}$, Krishnaswamy Ranganath ${ }^{4}$ \\ ${ }^{1}$ Department of Electronics and Communication Engineering, Gogte Institute of Technology, Belgaum, Karnataka, India \\ ${ }^{2}$ Vishwanathrao Deshpande Rural Institute of Technology, Haliyal, Karnataka, India \\ ${ }^{3}$ Retired, Indian Institute of Technology (Madras), Belgaum, Karnataka, India \\ ${ }^{4}$ RAGAVS Diagnostics and Research Center Pvt. Ltd., Bangalore, Karnataka, India
}

Email address:

supriya_sp@yahoo.com (S. S. Shanbhag)

To cite this article:

Supriya S. Shanbhag, Gururaj R. Udupi, K. Mothiram Patil, Krishnaswamy Ranganath. Quantitative Analysis of Diffusion Weighted MR Images of Intracerebral Haemorrhage by Signal Intensity Gradient Technique. International Journal of Medical Imaging.

Vol. 1, No. 1, 2013, pp. 12-18. doi: 10.11648/j.ijmi.20130101.13

\begin{abstract}
Intracerebral Haemorrhage (ICH) is an important public health problem leading to high rates of death and disability in adults. Early and reliable identification of the stage of ICH is vital when choosing the proper treatment and estimating patient's diagnosis and outcome. Diffusion Weighted - Magnetic Resonance Imaging (DW-MRI) presents a variation in the image signal intensity characteristics relative to the different stages of ICH and thus an increased understanding of the progression of the signal intensity variations on Diffusion Weighted (DW) images, subsequent to the onset of ICH, is valuable. In the present paper an effort was made to quantify the variations in the signal intensity characteristics on DW images, at evolving stages of ICH, for 32 subjects, by means of Signal Intensity Gradient (SIG) imaging metric. The relative increase in the SIG values (RSIG) for the subjects with ICH was in the range of $(3.83-35.67)$ times compared to their corresponding SIG values on the contralateral normal side. The observed RSIG values were elevated in Stage 1 (Hyperacute: $<1$ day) and further progressively decreased in Stage 2 (Acute: $1-7$ days) and Stage 3 (Late subacute: 7 - 14 days), and eventually reached their minimum in Stage 4 (Chronic: $>14$ days) of ICH. Also a negative correlation $(r=-$ 0.97) was observed between the RSIG values and the evolving stages of ICH. Therefore, the progression of the RSIG values could be supportive in understanding the developmental stages of ICH, and further be helpful in predicting the ICH stage and providing treatment at the appropriate time.
\end{abstract}

Keywords: Diffusion Weighted Images, Intracerebral Haemorrhage, Magnetic Resonance Imaging,

Signal Intensity Gradient

\section{Introduction}

Intracerebral Haemorrhage (ICH) accounts for $8-13 \%$ of all strokes and results from a wide spectrum of disorders [1]. Typically greater than one third of the patients with ICH do not survive and only twenty percent of the patients regain functional independence [1]. This high rate of morbidity and mortality has prompted investigations for new medical therapies for ICH $[1,2]$. However despite its relatively high incidence and poor associated outcomes, therapeutic advances for ICH remain limited. The limited success in establishing effective therapies is mainly due to the inadequate quantity and quality of clinical studies [3].
The radiological assessment of the subjects with suspected ICH is an extremely important part of their management. Traditionally Computed Tomography (CT) has been considered the gold standard to image ICH due to its high sensitivity and specificity. Nevertheless, studies carried out in the past indicate that Magnetic Resonance Imaging (MRI) has the capacity to show haemorrhages in different stages, enabling the assessment of bleeding onset, whereas CT is positive only for acute and subacute haemorrhages [4,5]. This information suggests that MRI should be the imaging modality of choice for subjects with ICH who may receive antithrombotic treatment [6].

The usual pattern of MRI appearances corresponding to the different stages of ICH is well known. The generalized 
model for the appearance of ICH on Magnetic Resonance (MR) images attributes the various signal intensity patterns of evolving ICH to the oxygenation state of hemoglobin, and the integrity of the red blood cells [7-9]. Even though conventional MRI has been frequently used to evaluate the appearance and underlying biophysical basis of evolving ICH over the past few years, Diffusion Weighted Magnetic Resonance Imaging (DW-MRI) has only recently been recognized as a valuable investigative resource [10-15]. DW-MRI makes use of additional strong diffusion-sensitizing gradients, and is extremely sensitive tool to monitor brain water diffusion changes with high temporal and spatial resolution, and therefore allows for more detailed insight into the pathophysiology of ICH [16].

Studies carried out in the past suggest that diffusion is restricted in the area of ICH before and after cell lysis, resulting in bright signal intensity on Diffusion Weighted (DW) images [7, 10]. Further, studies have been carried out to characterize haemorrhage on the basis of DW-MRI findings, and they qualitatively indicate that hematomas are initially hyper-intense on DW images and later hypo-intense during the first few days after onset $[10,11$, $17,18]$. Other investigators have recognized that hematoma signal intensity on DW images varies with time [7], but have not thoroughly quantified these signal intensity changes. Furthermore there are reports that describe the temporal evolution of Apparent Diffusion Coefficient (ADC) values within lesions following $\mathrm{ICH}$, to identify their usefulness in clinical diagnosis of $\mathrm{ICH}$ and treatment $[7,10,16,19,20]$.

Early and reliable detection of the stage of $\mathrm{ICH}$ is important for choosing the correct treatment and estimating patient's prognosis and outcome. Assessment of the stage of haemorrhage is important since it shows the exact state of the brain injury and thereby affects the rest of the therapy. In most of the clinical settings it is observed that radiologists access the $\mathrm{ICH}$ subjects only by observation of DW images, wherein, the hematoma signal intensity is visually graded as hyper-, iso, or hypo-intense, relative to the contralateral normal side of the subject $[7,10,18,21]$. This procedure requires high level of expertise in the field considering the complex parameters that affect the appearance of ICH on MRI. Therefore estimation by observation method is sensitive to experience and may be subjected to misinterpretation of the actual stage of haemorrhage. The objective of the present work was to examine the signal intensity characteristics of the various stages of ICH on DW images and realize a computer based method to automatically quantify the signal intensity variations at the evolving stages of $\mathrm{ICH}$. This information would definitely support the radiologists in the early and precise identification of the ICH stage and help out the further management and therapeutic decisions.

In the present work we have made an effort to grade the evolution of the signal intensity variations on DW images for the subjects with ICH using Signal Intensity Gradient (SIG) imaging parameter. Analysis was performed on a set of DW images of the human brain in the axial plane to find the relationship between the SIG values and the progression of $\mathrm{ICH}$, and present evidence in defining the stages of $\mathrm{ICH}$. The ADC methods reported in literature to study the signal characteristics of ICH on DW images generally use a dedicated workstation to record the ADC measurements by drawing Regions of Interests (ROIs) on the ADC map. For research purposes, using a separate workstation for measuring ADC values may not seem to be a laborious process, but it is an arduous and time-consuming task if measurements need to be obtained routinely. Further, calculation of the ADC values is done considering an ROI within the area of haemorrhage using a single axial section (showing ICH). For accurate analysis such methods demand for a thorough understanding of the entire haemorrhagic area as the ADC values may differ spatially within the area of haemorrhage [18].

The new method proposed in our work takes care of the drawbacks of the ADC methods that have been employed for the diagnosis of ICH. Our method most likely speeds up the investigation procedure as it can be carried out routinely without relying on a dedicated workstation and to some extent could also minimize the chances of inter-observer bias, as the entire area of haemorrhage is considered for image analysis. Further, all the axial sections showing ICH are taken into account to evaluate the SIG values, and for each axial section selected, the entire affected area (showing haemorrhage) is taken into consideration. Therefore, the method assures no loss of information as it covers larger area of the brain (multiple axial sections) and also rules out the demand on the need for expertise as the entire affected area is considered for carrying out the investigation. Overall, the main advantage of the proposed method is that the quantitative changes in the SIG values obtained from DW images might be assessed, and further they may possibly aid in the speedy and precise radiological identification of the ICH stage, as correct identification of the ICH stage is a critical step in planning the appropriate therapy.

\section{Materials and Methods}

\subsection{Subjects}

In our retrospective study, we investigated 32 intracerebral haemorrhagic lesions (22 male, 10 female, ages ranging from 28 years to 85 years with an average age of 57 years) of different stages using echo-planar DW-MRI. The appearance of ICH for all the 32 subjects was confirmed by ruling out the possibility of bright signal on DW images that is not due to restricted diffusion, but rather due to high T2 signal which "shines through" to the DW image, referred to as T2 shine through. This was done by verifying the appearance of hyper-intensities on DW images and concomitant reduced ADCs relative to the contralateral normal brain in their initial MR studies. Further all the subjects showed magnetic susceptibility 
artifact on gradient echo sequence, confirming it is haemorrhage. The ethics approval was obtained from the committee of clinical research at the RAGAVS Diagnostic and Research center, Bangalore, India, and Vikram Hospital, Bangalore, India.

The evolution of ICH can be described by five distinct stages, namely Stage 1: Hyperacute ( $<1$ day), Stage 2: Acute (1 - 3 days), Stage 3: Early subacute (3 - 7 days), Stage 4: Late subacute (7 - 14 days) and Stage 5: Chronic (>14 days) [9, 22]. However acute and early subacute stages of ICH display similar appearance on DW images [7, 13]. As a result, in the present work we have grouped the acute and early subacute stages of ICH collectively to form Stage 2, that is, the Acute stage (1 - 7 days). Accordingly in our work depending on the time interval between symptom onset and initial MRI, four stages were categorized. The four stages were: Stage 1: Hyperacute (ten subjects within 24 hours of symptom onset); Stage 2: Acute (five subjects between days 1 and 7); Stage 3: Late subacute (nine subjects between days 7 and 14) and Stage 4: Chronic (eight subjects after 14 days).

\subsection{Imaging Parameters}

All the subjects underwent clinical MR imaging with 1.5 T symphony maestro class MR scanning system from Siemens. DW-MRI was performed by using a multisection, single-shot, spin-echo, echo-planar pulse sequence with following parameters: Repetition Time [TR] $=3200 \mathrm{~ms}$, Echo Time $[\mathrm{TE}]=94 \mathrm{~ms}$, acquisition matrix $=128 \times 128$, Field of View [FOV] $=230 \mathrm{~mm} \times 230 \mathrm{~mm}$, and diffusion gradient value of $\mathrm{b}=1000 \mathrm{~s} / \mathrm{m}^{2}$ along 19 axial sections, 5 $\mathrm{mm}$ thick section and intersection gap of $1.5 \mathrm{~mm}$.

\subsection{Signal Intensity Gradient}

DW-MRI is highly sensitive to changes in water motion [23], because signal loss occurs with more water diffusion when using a diffusion-sensitized pulse sequence. The presence of intact cell membranes restricts molecular diffusion [24] and the lysis of RBC membranes in the evolution of hematomas might, therefore, change the environment regarding water mobility [10]. DW images thereby differ among the stages of evolving hematomas in that the appearance of signal intensities on DW images transforms from being hyper-intense to hypo-intense as the haemorrhage evolves with time, from one stage to another [7, 10, 18]. Accordingly the spatial intensity variation distribution of the subjects with ICH on DW images reveals that the image signal intensity is not uniformly distributed over the entire DW image (high signal intensity in the area of haemorrhage). There are abrupt jumps in the image signal intensity in the areas of haemorrhage in contrast to the other healthy areas of the brain, where the signal intensity distribution is almost uniform [25, 26]. We have made an attempt to investigate and quantify the water molecule diffusion patterns producing signal intensity variations in the different stages of ICH using the SIG parameter. The strength of the signal intensity variations quantified by the SIG values would thereby provide information about the brain tissue architecture, either normal or in a diseased state. To obtain the relative SIG values (RSIG), the SIG values evaluated in the area of haemorrhage were compared to the corresponding SIG values from the contralateral side of the same subject using (1).

$$
\text { RSIG }=\frac{\text { SIG on ICH side }- \text { SIG on contralateral side }}{\text { SIG on contralateral side }}
$$

The RSIG values so obtained were employed in differentiating the haemorrahgic tissues from the healthy tissues, and further employed in the categorization of the different stages of ICH. Consequently, detection and quantification of the observed signal intensity variations on DW images, in the areas of brain showing ICH, may allow for the characterization of the ICH stages and by this means aid in the proper planning of the clinical treatment.

\subsection{Data Analysis}

DW images obtained in DICOM format were converted to bmp format with intensities scaled to fit the conventional range of $(0-255)$. This was done to reduce the complexity in the image manipulation algorithms and to achieve speed up in processing the images. DW image obtained from each $\mathrm{ICH}$ subject in the axial plane was divided into six anatomically significant areas as shown in Fig.1.

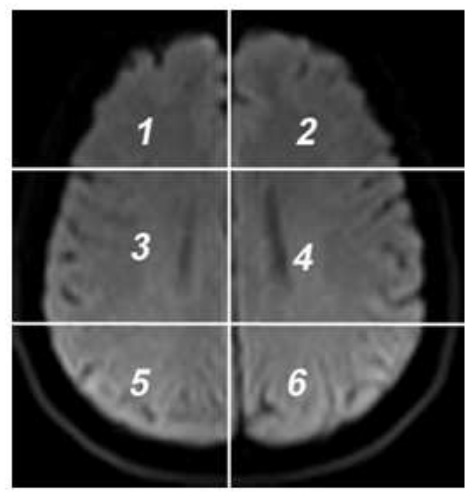

Figure 1. Axial DW image showing areas of brain

For each subject with $\mathrm{ICH}$, we obtain a set of DW images in the axial plane taken at different axial sections (from 1 to 19). The subject with ICH presents abrupt changes in the image signal intensities in one (or more) of the six areas, in the affected axial sections. The axial sections that indicate such changes in the signal intensities were selected for image analysis. For each axial section selected from the ICH subject, the following procedure was employed to obtain the decisive SIG value for that subject. Firstly an ROI, $I(x, y)$, was manually placed in the particular area of the DW image, covering the high intensity region showing $\mathrm{ICH}$ for that axial section. An equivalent (mirror) ROI of the same size was manually 
selected on the contralateral normal side of the same subject for comparison. The ROIs on each side were further divided automatically (using Adobe Photo-shop CS3) into smaller sub-regions, $f(x, y)$, corresponding to an image size represented by $(\mathrm{M} \times \mathrm{N})$ pixels. The typical values for $\mathrm{M}$ and $\mathrm{N}$ selected for image analysis range in between 16 to 18 pixels. Additional care was taken while choosing the sub-regions to avoid any errors that may be caused due to edge effects (change in intensity along the periphery of ICH). The ROI and the corresponding sub-regions on the $\mathrm{ICH}$ side and on the contralateral normal side for a subject with ICH (area 1, axial section 16) are shown in Fig.2.

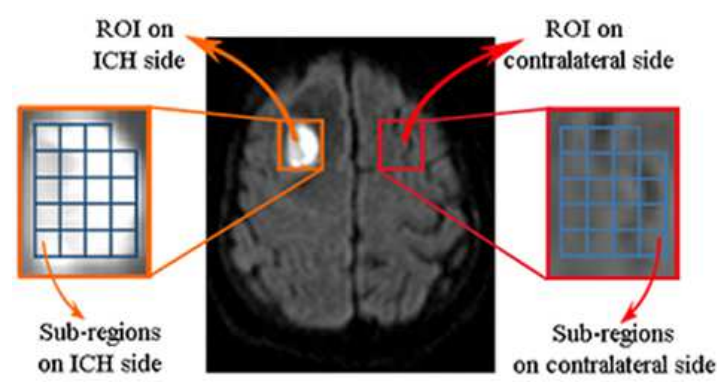

Figure 2. DW image showing $R O I(I(x, y))$ and sub-regions $(f(x, y))$ on ICH side (area 1, axial section 16) and contralateral side

Given that water diffusion has directionality, in order to take care of the orientation dependent contrast (diffusion anisotropy), the SIG value for each sub-region, $f(x, y)$, was evaluated using the Robinson compass operator comprising of eight kernels as shown in Fig.3. The Robinson method is easier to implement because it relies only on coefficients of 0,1 and 2, and the masks are symmetrical about their directional axis (axis with the zeros). The set of 8 kernels applied in Robinson's operator are produced by taking one kernel and rotating its coefficients circularly [27]. These kernels were convolved with the input image, $f(x, y)$, to obtain the SIGs along the corresponding directions. GS, GSE, GE, GNE, GN, GNW, GW, and GSW, represents gradients along South, South-East, East, North-East, North, North-West, West and South-West directions respectively. The magnitude of the maximum change in the SIG is the maximum value gained from applying all the eight kernels to the pixel neighborhood. The maximum SIG value, represented as $\mathrm{SIG}_{\max }$, is given by (2) [25, 27].

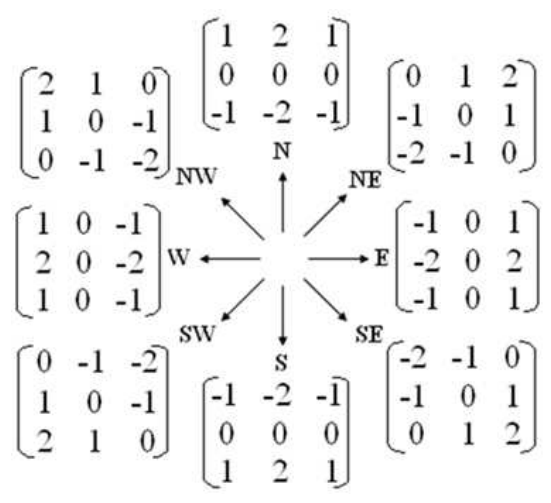

Figure 3. Robinson kernels and their orientation
SIGmax $=\max \{\mathrm{GS}, \mathrm{GSE}, \mathrm{GE}, \mathrm{GNE}, \mathrm{GN}, \mathrm{GNW}, \mathrm{GW}, \mathrm{GSW}\}$

For each sub-region on the ICH side, the magnitude and the location of $\mathrm{SIG}_{\max }$ was evaluated. Subsequently, $\mathrm{SIG}_{\max }$ values obtained from all the sub-regions of the ROI on the ICH side were scanned to obtain the value of the overall maximum SIG value for that axial section. The corresponding SIG value from the contralateral normal side was noted for comparison. The magnitude of the maximum SIG value so obtained for the axial section indicates whether there is any sudden change in the signal intensity level, in any of the sub-regions of the ROI for that axial section. Further, if there is any such change observed, we can readily establish the sub-region, and also the exact location ( $(\mathrm{x}, \mathrm{y})$ coordinates) within that sub-region.

The process was repeated for all the axial sections selected for image analysis from the ICH subject. After obtaining the respective maximum SIG values for all the axial sections, the decisive SIG value was chosen as the highest value of the maximum SIG values obtained across all the selected axial sections. The equivalent SIG value on the contralateral normal side was noted for comparison. The procedure was repeated for all the ICH subjects considered under study and finally, the RSIG values were evaluated.

\subsection{Statistical Analysis}

Statistical analysis was carried out using excel program and SPSS 17.0 software package. The results were tested for statistical significant difference between the SIG values obtained for the subjects with ICH and their contralateral normal side. Similarly the statistical significant difference between the RSIG values obtained for the subjects with ICH across the different stages was tested. The analysis was carried out at $99 \%$ confidence level $(\mathrm{p}<0.01)$ with $\mathrm{z}$ test, using the significant difference of means method [28]. The correlation between the RSIG values and the different stages of ICH was evaluated.

\section{Results and Discussion}

The DW image of a subject showing ICH in area 3, axial section 11 is shown in Fig. 4.

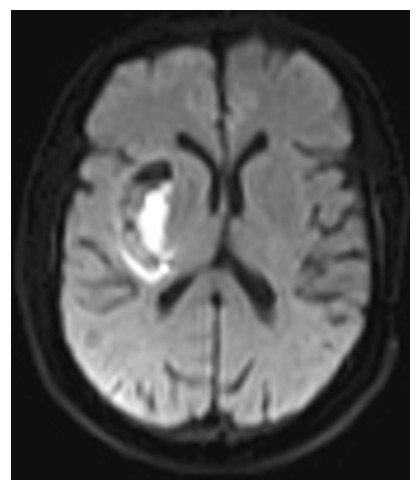

Figure 4. Axial DW image showing ICH in area 3, axial section 11 
The plot of the spatial variation of intensity distribution for a sub-region of the ROI in area 3, axial section 11, resulting in maximum SIG value for the ICH subject is shown in Fig.5. The corresponding plot of the spatial variation of intensity distribution for the sub-region on the contralateral side is shown in Fig.6.

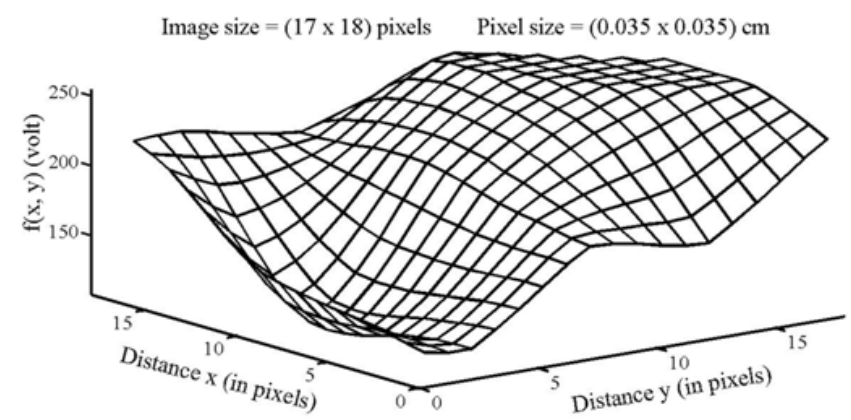

Figure 5. Spatial variation of image intensity distribution of ICH subject in area 3, axial section 11

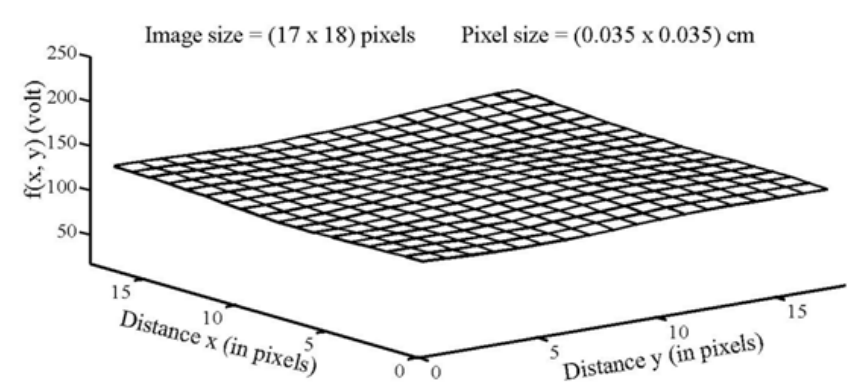

Figure 6. Spatial variation of image intensity distribution on the contralateral side

It is observed from Fig.5 that the spatial intensity variation distribution for the subject with $\mathrm{ICH}$ has abrupt jumps (non-uniform) and this leads to higher value for the maximum SIG as compared to that on the contralateral side, where in, the spatial intensity variation distribution is almost uniform. Consequently maximum SIG value on the haemorrhage side (252) is much elevated compared to the corresponding SIG value on the contralateral side (26). The maximum SIG value evaluated indicates the greatest change in the image signal intensity variation across the selected ROI in area 3, axial section 11, and also indicates its exact location, $(11,1)$, in the (x, y) co-ordinates (Fig.5).

The semilog plot of the variation in the decisive SIG value in the area of haemorrhage and the corresponding SIG value on the contralateral side, for all the 32 subjects considered in our study is shown in Fig.7. The difference in the decisive SIG values for the subjects with ICH compared to their contralateral side were highly significant $(\mathrm{p}<0.01)$ in the areas of the brain where there was an occurrence of haemorrhage. The results suggest that the decisive SIG values obtained from DW images for the subjects with ICH can be used to clearly differentiate them from the normal subjects.

The range of the decisive SIG values in the area of haemorrhage and on the contralateral side, as well as the

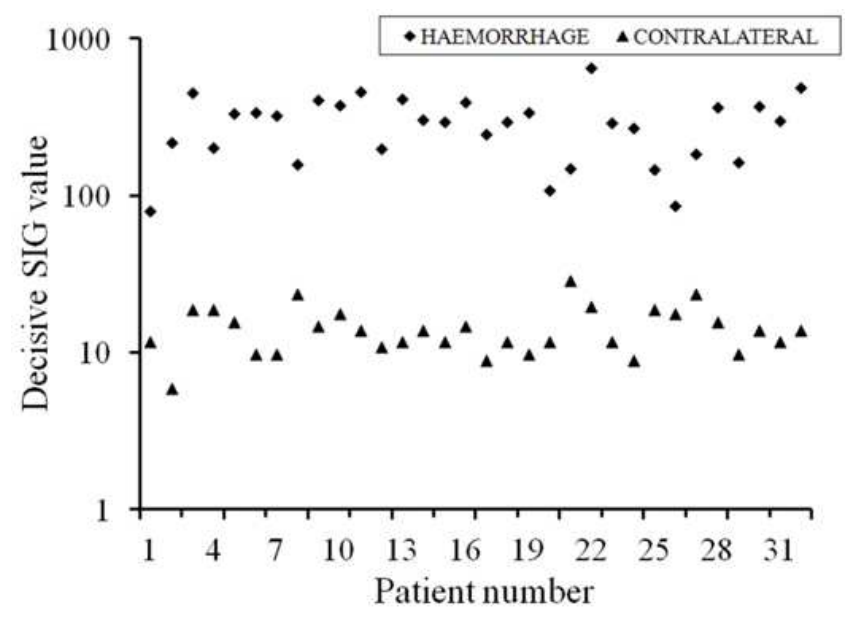

Figure 7. Variation in the decisive SIG values (semilog plot) on haemorrhage side and the corresponding $S I G$ values on the contralateral side

mean RSIG values for the subjects in each time stage of haemorrhage is shown in Table 1 . The variation in the mean RSIG values for each set of subjects within the different time stages of ICH (last column of Table 1) is plotted in Fig.8. It is observed that the RSIG values are notably higher for Stage 1: Hyperacute ( $<1$ day) of $\mathrm{ICH}$ as compared to all the other three stages. There is a subsequent decline in the RSIG values as the haemorrhage lesions evolve from Stage 2: Acute (1 - 7 days) to Stage 3: Late subacute ( 7 - 14 days). The Stage 4: Chronic (>14 days) presents the lowest values for the RSIG parameter. Further there was no overlap observed between the RSIG values obtained across the four stages of $\mathrm{ICH}$. Therefore we quantitatively confirm that the appearance of ICH is mainly hyper-intense on DW images in Stage 1: Hyperacute $(<1$ day) resulting in high values of RSIG and the progression into Stage 2: Acute (1 - 7 days) and Stage 3: Late subacute ( 7 - 14 days) is identified by a fall in the signal intensity and corresponding lowering of the RSIG values. The Stage 4: Chronic ( $>14$ days) shows marked hypo-intensity and thereby results in the lowest values of RSIG. There was a significant difference $(p<0.01)$ observed between the RSIG values across the different stages of $\mathrm{ICH}$.

Table 1. Decisive SIG and mean RSIG values in different time stage of ICH

\begin{tabular}{lllll}
\hline $\begin{array}{l}\text { ICH stage } \\
\text { (days) }\end{array}$ & $\begin{array}{l}\text { Number } \\
\text { of Subjects }\end{array}$ & \multicolumn{2}{l}{ Decisive SIG values } & Mean RSIG \\
\hline $\begin{array}{l}\text { Hyperacute } \\
(<1)\end{array}$ & 10 & $220 \sim 649$ & $6 \sim 20$ & Contralateral \\
$\begin{array}{l}\text { Acute } \\
(1-7)\end{array}$ & 5 & $297 \sim 411$ & $12 \sim 15$ & $25.09 \pm 1.10$ \\
$\begin{array}{l}\text { Late subacute } \\
(7-14)\end{array}$ & 9 & $163 \sim 378$ & $10 \sim 19$ & $20.58 \pm 2.81$ \\
$\begin{array}{l}\text { Chronic } \\
(>14)\end{array}$ & 8 & $80 \sim 202$ & $19 \sim 32$ & $6.29 \pm 1.92$ \\
\hline
\end{tabular}




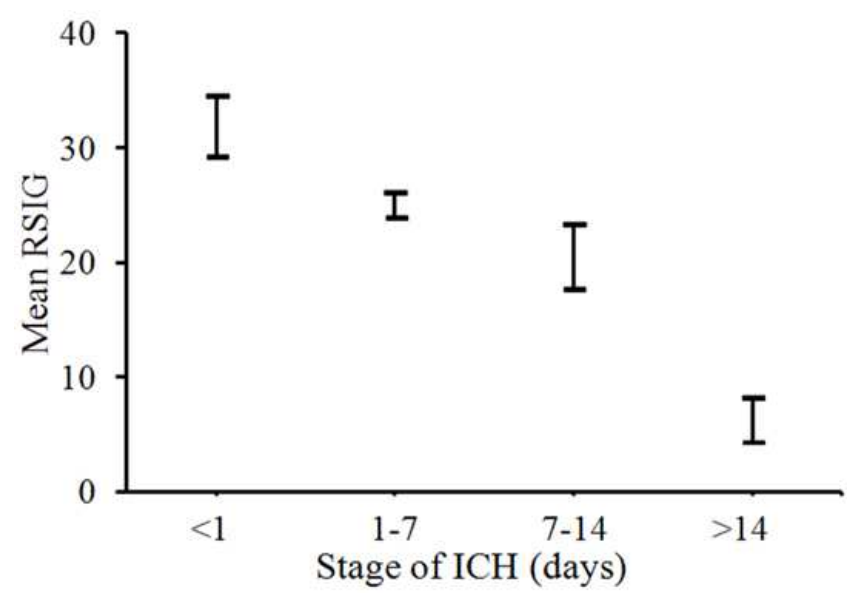

Figure 8. Variation in the mean RSIG values with the stage of ICH

The plot of the variation in the RSIG values with the different stages of ICH for all the 32 subjects considered in our study is shown in Fig.9. The line given by the equation $\mathrm{y}=-0.04 * \mathrm{x}+31.99$, represents the linear regression that fit the data. A negative correlation of the order of $r=-0.97$ was found between the RSIG values and the different stages of $\mathrm{ICH}$, which is an indicator that early stages of ICH gives rise to higher values of RSIG. The evolution of the RSIG values observed subsequent to $\mathrm{ICH}$ is suggestive that they can be supportive in understanding the developmental stages of ICH and can positively assist in classifying the stages of $\mathrm{ICH}$.

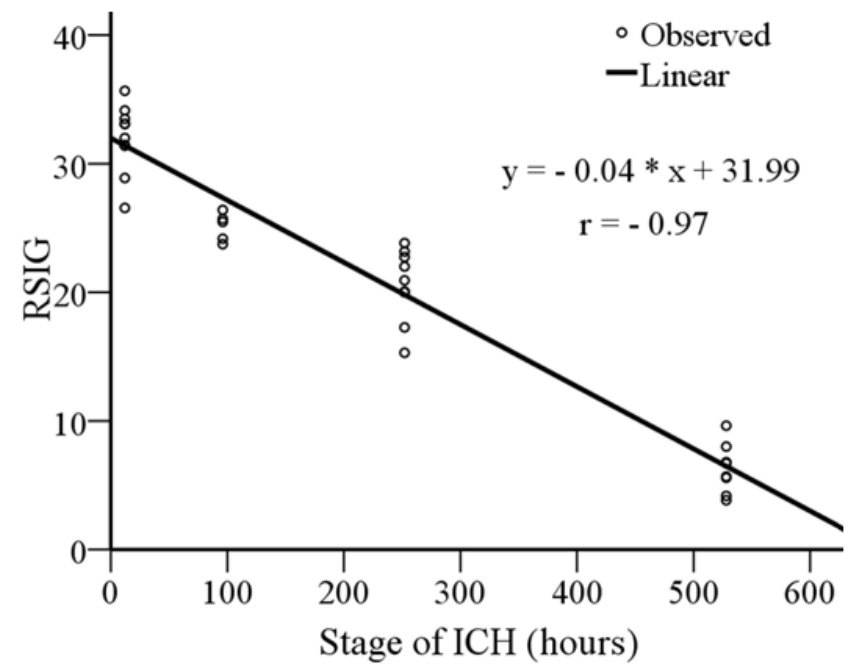

Figure 9. Variation in the RSIG values with the stage of ICH

Our study supports the findings of the previously published work $[7,9,18]$ which propose that the signal intensity characteristics on DW images of the brain undergo a series of variations after the onset of ICH. Further it provides evidence that the quantification of the signal intensity variations on DW images using RSIG values could be effectively employed in the characterization of the stages of ICH. However unlike the methods using ADC values $[10,17]$, where, only a small ROI on a particular axial section from the ICH subject was considered for investigation, we have included the entire area of haemorrhage on all the axial sections that show hyperintensity in order to arrive at the final RSIG value.

\section{Conclusion}

The present study showed that the SIG and RSIG values change temporally in the haemorrhagic lesions of the brain demonstrating the evolution of ICH. The SIG values obtained from DW images can be used to noticeably differentiate the haemorrhagic tissues from the normal tissues. The range of the RSIG values for the ICH subjects was found to be from $(3.83-35.67)$. The progression of the RSIG values after the onset of haemorrhage shows that they can be assessed and probably used to identify the different stages of $\mathrm{ICH}$, so that early changes taking place in the brain can be detected. Therefore, the results in our study signify that the adoption of the RSIG values in the clinical diagnosis of ICH could be supportive and instructive in the progression and treatment of $\mathrm{ICH}$. This could positively assist the medical personnel in determining the severity of the brain haemorrhage, to consider early remedial methods.

\section{Acknowledgements}

The authors would like to thank Dr. Sudhir Pai (Sr. Consultant, Neurosurgeon, Vikram Hospital, Bangalore, India) for his valuable assistance in data collection and Dr. Deepak Y. S. (Consultant Radiologist, Bangalore, India) for his helpful and constructive suggestions during the planning and development of this research work.

\section{References}

[1] C. Counsell, S. Boonyakarnkul, M. Dennis, P. Sandercock, J. Bamford, J. Burn, C. Warlow, "Primary intracerebral haemorrhage in the Oxfordshire community stroke project," Cerebrovascular Diseases, vol. 5, no. 1, pp. 26-34, 1995.

[2] A. I. Qureshi, Y. M. Mohammad, A. M. Yahia, J. I. Suarez, A. M. Siddiqui, J. F. Kirmani, M. F. Suri, J. Kolb, O. O. Zaidat, "A prospective multicenter study to evaluate the feasibility and safety of aggressive antihypertensive treatment in patients with acute intracerebral hemorrhage," Journal of Intensive Care Medicine, vol. 20, no. 1, pp. 34-42, Jan.-Feb. 2005.

[3] J. P. Broderick, H. P. Adams Jr, W. Barsan, W. Feinberg, E. Feldmann, J. Grotta, C. Kase, D. Krieger, M. Mayberg, B. Tilley, J. M. Zabramski, M. Zuccarello, " Guidelines for the management of spontaneous intracerebral hemorrhage. A statement for healthcare professionals from a special writing group of the stroke council. American Heart Association," Stroke, vol. 30, no. 4, pp. 905-915, Apr. 1999.

[4] C. S. Kidwell, J. L. Saver, J. P. Villablanca, G. Duckwiler, A. Fredieu, K. Gough, M. C. Leary, S. Starkman, Y. P. Gobin, R. Jahan, P. Vespa, D. S. Liebeskind, J. R. Alger, F. Vinuela, "Magnetic resonance imaging detection of microbleeds before thrombolysis: an emerging application," Stroke, vol. 33, no. 1, pp. 95-98, Jan. 2002. 
[5] N. Nighoghossian, M. Hermier, P. Adeleine, K. Blanc-Lasserre, L. Derex, J. Honnorat, F. Philippeau, J.F. Dugor, J. C. Froment, P. Trouillas, "Old microbleeds are a potential risk factor for cerebral bleeding after ischemic stroke : a gradient-echo T2*-weighted brain MRI study," Stroke, vol. 33, no. 3, pp. 735-742, Mar. 2002.

[6] R. von Kummer, "MRI: The new gold standard for detecting brain hemorrhage?," Stroke, vol. 33, no. 7, pp. 1748-1749, Jul. 2002.

[7] B. K. Kang, D. G. Na, J. W. Ryoo, H. S. Byun, H. G. Roh, Y. S. Pyeun, "Diffusion-weighted MR imaging of intracerebral haemorrhage," Korean Journal of Radiology, vol. 2, no. 4, pp. 183-191, Oct.-Dec. 2001.

[8] J. M. Gomori, R. I. Grossman, H. I. Goldberg, R. A. Zimmerman, L. T. Bilaniuk, "Intracranial hematomas: imaging by high-field MR," Radiology, vol. 157, no. 1, pp. 87-93, Oct. 1985.

[9] W. G. Bradley, "MR appearance of hemorrhage in the brain," Radiology, vol. 189, no. 1, pp. 15-26, Oct. 1993.

[10] S. W. Atlas, P. DuBois, M. B. Singer, D. Lu, "Diffusion measurements in intracranial hematomas: implications for MR imaging of acute stroke," American Journal of Neuroradiology, vol. 21, no. 7, pp. 1190-1194, Aug. 2000.

[11] T. Ebisu, C. Tanaka, M. Umeda, M. Kitamura, M. Fukunaga, I. Aoki, H. Sato, T. Higuchi, S. Naruse, Y. Horikawa, S. Ueda "Hemorrhagic and nonhemorrhagic stroke: diagnosis with diffusion-weighted and T2-weighted echo-planar MR imaging," Radiology, vol. 203, no. 3, pp. 823-828, Jun. 1997.

[12] P. D. Schellinger, O. Jansen, J. B. Fiebach, W. Hacke, K. Sartor, "A standardized MRI stroke protocol: comparison with CT in hyperacute intracerebral hemorrhage," Stroke, vol. 30, no. 4, pp. 765-768, Apr. 1999.

[13] S. Felber, A. Auer, C. Wolf, M. Schocke, S. Golaszewski, B. Amort, D. zurNedden, "MRI characteristics of spontaneous intracerebral hemorrhage," Radiologe, vol. 39, no. 10, pp. 838-846, Oct. 1999.

[14] M. Wiesmann, T. E. Mayer, I. Yousry, G. F. Hamann, H. Bruckmann, "Detection of hyperacute parenchymal hemorrhage of the brain using echo-planar T2*-weighted and diffusion-weighted MRI," European Radiology, vol. 11, no. 5, pp. 849-853, 2001

[15] J. R. Carhuapoma, P. Y. Wang, N. J. Beauchamp, P. M. Keyl, D. F. Hanley, P. B. Barker, "Diffusion-weighted MRI and proton MR spectroscopic imaging in the study of secondary neuronal injury after intracerebral hemorrhage," Stroke, vol. 31, no. 3, pp. 726-732, Mar. 2000.

[16] S. Attia, G. Mohamed, El Khatib, M. Bilal, H. Nassar, "Spontaneous intracerebral hematoma in young people: clinical and radiological magnetic resonance imaging features by diffusion-weighted images," Egyptian Journal of Neurology, Psychiatry and Neurosurgery, vol. 44, no. 2, Jul. 2007.
[17] T. Hideichi, K. Masahito, S. Sadao, S. Fumiko, M. Ban, "Cerebral haemorrhage. Serial study using diffusion-weighted MRI," Japanese Journal of Stroke, vol. 21, no. 2, pp. 245-252, 1999.

[18] S. Silvera, C. Oppenheim, E. Touzé, D. Ducreux, P. Page, V. Domigo, J. L. Mas, F. X. Roux, D. Frédy, J. F. Meder, "Spontaneous intracerebral hematoma on diffusion-weighted images: influence of T2-shine through and T2-blackout effects," American Journal of Neuroradiology, vol. 26, no. 2, pp. 236-241, Feb. 2005.

[19] T. W. Stadnik, P. Demaerel, R. R. Luypaert, C. Chaskis, K. L. Van Rompaey, A. Michotte, M. J. Osteaux, "Imaging tutorial: differential diagnosis of bright lesions on diffusion-weighted MR images,” Radiographic, vol. 23, no. 1, Jan. 2003.

[20] J. A. Maldjian, J. Listerud, G. Moonis, F. Siddiqi, "Computing diffusion rates in T2-dark hematomas and areas of low T2 signal," American Journal of Neuroradiology, vol. 22, no. 1, pp. 112-118, Jan. 2001.

[21] I. Linfante, R. H. Llinas, L. R. Caplan, S. Warach, "MRI features of intracerebral hemorrhage within 2 hours from symptom onset," Stroke, vol. 30, no. 11, pp. 2263-2267, Nov. 1999.

[22] J. A. Chalela, C. S. Kidwell, L. M. Nentwich, M. Luby, J. A. Butman, A. M. Demchuk, M. D. Hill, N. Patronas, L. Latour, S. Warach, "Magnetic resonance imaging and computed tomography in emergency assessment of patients with suspected acute stroke: a prospective comparison," The Lancet, vol. 369, no. 9558, pp. 293-298, Jan. 2007.

[23] D. Chien, R. B. Buxton, K. K. Kwong, T. J. Brady, B. R. Rosen, "MR diffusion imaging of the human brain," Journal of Computer Assisted Tomography, vol. 14, no. 4, pp. 514-520, Jul.1990.

[24] J. W. van der Veen, P. van Gelderen, J. H. Creyghton, W. M. Bovee, "Diffusion in red blood cell suspensions: separation of the intracellular and extracellular NMR sodium signal," Magnetic Resonance in Medicine, vol. 29, no. 4, pp. 571-574, Apr. 1993.

[25] R. C. Gonzalez and P. Wintz, Image transforms in 'Digital Image Processing'. $2^{\text {nd }}$ edition, Addison-Wesley, U.S.A, 1987.

[26] K. G. Prabhu, K. M. Patil, S. Srinivasan, "Diabetic feet at risk: a new method of analysis of walking foot pressure images at different levels of neuropathy for early detection of plantar ulcers," Medical and Biological Engineering and Computing, vol. 39, no. 3, pp. 288-293, 2001.

[27] K. C. Sudeep, J. A. Majumdar, "Novel architecture for real time implementation of edge detectors on FPGA," International Journal of Computer Science Issues, vol. 8, no. 1, pp. 193-202, Jan. 2011.

[28] S. C. Gupta, V. K. Kapoor, Fundamentals of mathematical statistics. $11^{\text {th }}$ edition, Sultan Chand and Sons, New Delhi, India, 1982 